\title{
Diskussion optionaler Maßnahmen zur Maximierung des effektiven Durchsatzes von automatischen Zeilenregallagern nach dem Ausfall einer Gasse
}

\author{
Dipl.-Ing. Christian Wildner \\ Technische Universität IImenau \\ Fakultät für Maschinenbau \\ Fachgebiet Fabrikbetrieb
}

\begin{abstract}
Automatische Zeilenregallager und insbesondere Hochregallager werden weithin unter Bedingungen betrieben, die nach dem Ausfall einer Gasse eine deutliche Minderung des maximalen effektiven Durchsatzes des Lagers bewirken [Gei98].
\end{abstract}

Dieser Beitrag nennt einführend die relevanten Betriebsbedingungen [Wil10] und konkretisiert mittels geeigneter Experimente an Ereignis-orientierten, diskreten Simulationsmodellen den Einfluss dieser Bedingungen auf die Ausprägung des betrachteten Effektes.

Darüber hinaus beschreibt und diskutiert dieser Beitrag neben den bisher genannten [Sch10] [Wil10] weitere optionale Maßnahmen unter Einbeziehung von Mitteln der Simulation, die geeignet sind, der Minderung des maximalen effektiven Durchsatzes entgegen zu wirken. Ziel ist es hierbei, vor dem Hintergrund spezifischer Einsatzsituationen die Auswahl geeigneter Optionen (ggf. auch in Kombination) und somit auch eine spezifische Konfiguration der besprochenen Maßnahmen im Sinne einer auf den jeweiligen Anwendungsfall abgestimmten Anlaufstrategie zu ermöglichen.

\section{Betriebsziele und -einflüsse}

Lager werden zweckgerichtet betrieben - der Betrieb eines Lagers hat sich an spezifischen Zielen zu orientieren. Die Anwendung von Strategien dient im Lagerbetrieb dazu, diese Ziele weitestgehend zu erfüllen. Strategien können in diesem Zusammenhang allgemein als Wege zur Zielerfüllung aufgefasst werden, als Entscheidungsregeln für oder gegen unterschiedliche Handlungsalternativen [Mar06] - ihre Umsetzung bewirkt allgemein eine sukzessive Einschränkung des Lösungsfeldes [Bra97].

Marktwirtschaftliche Entwicklungstendenzen wirken sich in Form von Anforderungsänderungen auch auf Lager aus. Neben dem Trend zur Flexibilisierung lässt sich hierbei auch generell eine immer weiter steigende Auftragslast konstatieren [tSN07]. Steigende Auftragslasten bedingen die Anforderung nach einer Minimierung der Auftragsdurchlaufzeit [tS08] oder anders ausgedrückt: nach einer Maximierung des Durchsatzes. 
Zur Verfolgung des Ziels der Durchsatzmaximierung bietet sich in automatischen Zeilenregallagern mit gassengebundenen Regalbediengeräten die Anwendung mehrerer Strategien an, die sich je nach Einsatzsituation zu einem Optimum kombinieren lassen (u.a. [Bra97], [Gra94], [Sch08]).

Unter Berücksichtigung der sog. Qualitätsziele logistischer Leistungssysteme [Gud10] sollte man in diesem Zusammenhang darüber hinaus auch von steigenden Anforderungen insbesondere hinsichtlich der Leistungsbereitschaft und der Termintreue allgemein ausgehen können. Hieraus ergeben sich nicht nur erhöhte Anforderungen nach Einhaltung vorgegebener Zeitfenster der Auftragsbearbeitung [tS08], sondern auch erhöhte Anforderungen an die Lieferfähigkeit im Besonderen. Um die Lieferfähigkeit in der Planung von Lagern zu berücksichtigen, ist neben dem Sicherheitsbestand auch die Unterbrechungsreserve von Bedeutung [Gud06]. Letztere - auch als "eiserner Bestand“ bezeichnet - dient in diesem Zusammenhang dazu, die Versorgung der Verbrauchsstelle auch für die Dauer eines Anlagenausfalls sicherzustellen [Ker96].

Ein Maximum an Zugriffssicherheit auf die Einheiten eines Lagers leistet einen wichtigen Beitrag zur Lieferfähigkeit des Logistiksystems. Um maximale Zugriffssicherheit weitestgehend zu realisieren, existieren zur sog. Querverteilungsoder Gleichverteilungsstrategie keinerlei Alternativen. Diese sieht die Verteilung des Lagerbestands eines Artikels auf mehrere Lagergassen vor: Fällt eine Lagergasse vorübergehend aus und ist der Lagerbestand des nachgefragten Artikels größer eins, existiert in mindestens einer weiteren Lagergasse eine entsprechende Lagereinheit, auf die ein Zugriff weiterhin möglich ist. Zudem ermöglicht diese Strategie einen höheren Durchsatz bei gleichzeitiger Nachfrage mehrerer Lagereinheiten im ungestörten Lagerbetrieb desselben Artikels, da diese dann auch aus mehreren Gassen parallel ausgelagert werden können.

Die Strategie FIFO (first-in-first-out) steht in keinem direkten Bezug zu den beiden genannten Betriebszielen. Sie ist ursprünglich - neben möglicherweise relevanten baulich-konstruktiven Rahmenbedingungen - mit Blick auf das Qualitätsziel der Sendungsqualität, genauer: hinsichtlich der Qualitätsmerkmale Unversehrtheit und Mängelfreiheit [Gud10] speziell für die einem Verfallsdatum unterliegenden Lagergütern (Mindesthaltbarkeit) eingeführt worden und wurde in der Folge auch bei allen anderen gefordert [Gra93].

Die Anwendung von Querverteilungsstrategie und FIFO erscheint zudem offensichtlich als standardmäßig, in ihrer Wirkung ausreichend überschaubar und durchweg positiv, dass sie im Rahmen von Vorhaben der Lagerplanung seitens externer Auftraggeber gesondert eingefordert werden [Gud10].

Der kombinierte Einsatz eben dieser beiden Strategien Querverteilung und FIFO hat allerdings auf den ersten Blick schwer vorhersehbare negative Auswirkungen auf bestimmte Phasen des Betriebes automatischer Zeilenregallager mit gassengebundenen Regalbediengeräten. 


\section{Situation der Wiederinbetriebnahme einer ausgefallenen Gasse}

Auftretende Störungen können - wenn ein automatisches Zeilenregallager ausschließlich über gassengebundene Regalbediengeräte verfügt - dazu führen, dass einzelne Gassen vorübergehend nicht befahren werden können, d.h. dass aus den beiden Regalen einer betroffenen Gasse weder ein- noch ausgelagert werden kann. Findet in einem Lager dieser Art zudem sowohl die Querverteilungsstrategie als auch das FIFO-Prinzip üblicherweise - also auch während eines Gassenausfalls - Anwendung, so entwickeln sich im Verlauf der Dauer eines Gassenausfalls unweigerlich Ungleichgewichte in der Verteilung der Einheiten über die Lagergassen.

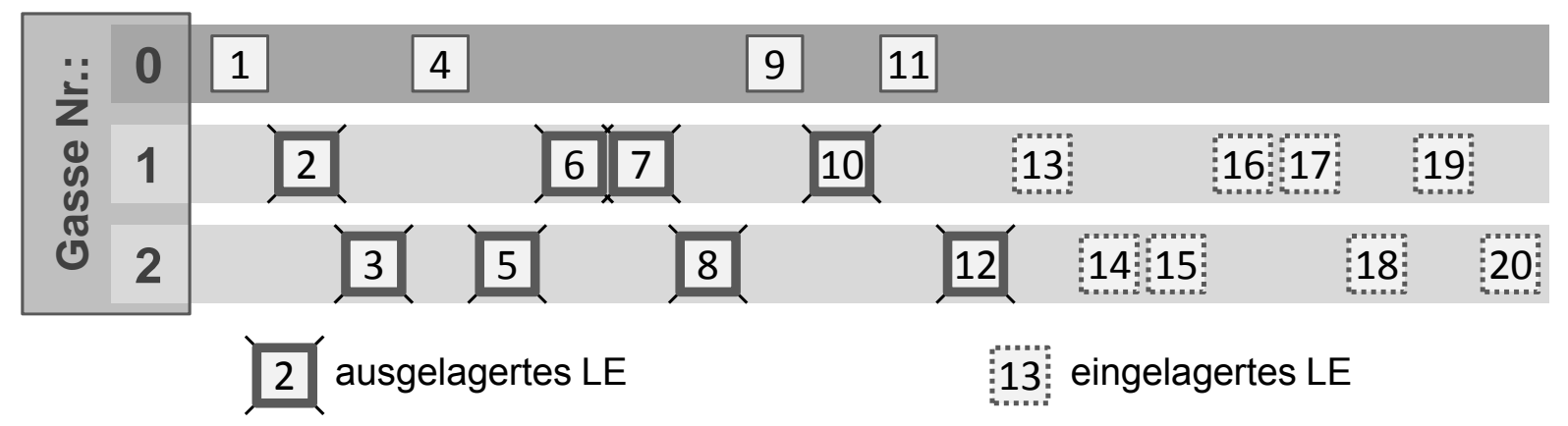

Abbildung 1: sortenspezifische Zu- und Abgänge während eines Gassenausfalls

In Abbildung 1: „sortenspezifische Zu- und Abgänge während eines Gassenausfalls“ [Wil10] ist die Verteilung von Lagereinheiten (LE) einer Artikelsorte vor und nach dem Ausfall der Gasse Nr. 0 in einem dreigassigen Zeilenregallager dargestellt. Mit den Nummern 1 bis 12 sind diejenigen LE bezeichnet, die vor dem Gassenausfall eingelagert worden waren. Während des Ausfalls bestand keine Möglichkeit, Einheiten in Gasse 0 einzulagern oder von dieser auszulagern. Die acht im Zuge der Ausfallsdauer einzulagernden LE werden deshalb in die Gassen 1 und 2 verteilt (LE Nummer 13 bis 20). Diesen beiden Gassen entnommen werden die im selben Zeitraum nach dem FIFO-Prinzip auszulagernden LE Nr. 2, 3, 5, 6, 7, 8, 10 und 12 der betrachteten Sorte. Die LE 1 und 4 können aufgrund des Ausfalls der Gasse 0 nicht beachtet werden. (Im ungestörten Betrieb wären eigentlich die LE 1 bis 8 ausgelagert worden.)

Im gewählten Beispiel ergibt sich zum Zeitpunkt der Wiederinbetriebnahme der ausgefallenen Gasse 0 eine Bestandssituation, wie sie in Abbildung 2: „sortenspezifische Gassenbestände bei Wiederinbetriebnahme" dargestellt ist.

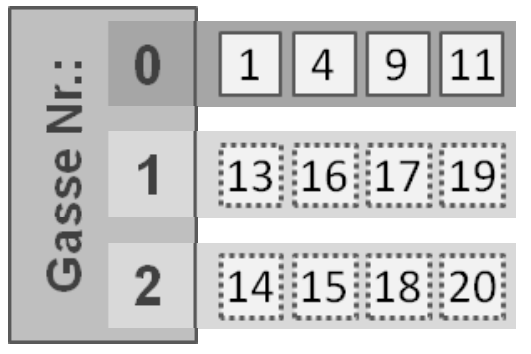

Abbildung 2: sortenspezifische Gassenbestände bei Wiederinbetriebnahme 
In jeder der drei Gassen befinden sich vier LE der betrachteten Sorte - diese Verteilung von LE entspricht damit aber nur scheinbar dem Ergebnis einer Querverteilung: Denn anders als tatsächlich über alle Lagergassen quer- bzw. gleichverteilte Gassenbestände, unterliegen die in Abbildung 2 dargestellten LE einer eigenen, besonderen Reihenfolge, wenn entsprechend viele Auslageraufträge der betrachteten Sorte auszuführen sind.

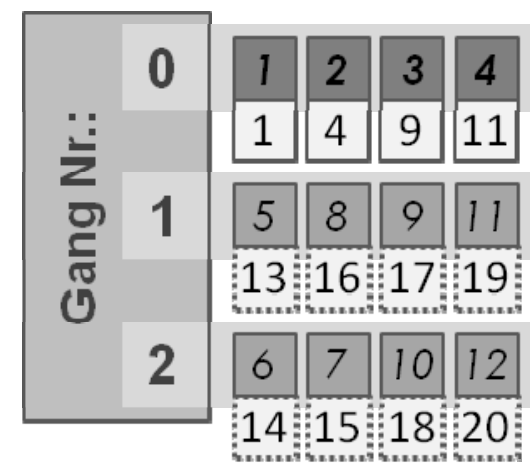

Abbildung 3: Auslagerreihenfolge nach Wiederinbetriebnahme

In Abbildung 3: „Auslagerreihenfolge nach Wiederinbetriebnahme“ sind die sortenspezifischen Gassenbestände aus Abbildung 2 entsprechend ihrer FIFOAuslagerreihenfolge nummeriert (Zahlen in grau-gefüllten Kästchen) - wobei offensichtlich wird, dass die LE 1, 4, 9 und 11 die ersten vier auszulagernden LE darstellen.

Abbildung 4: "Algorithmus der Auftragsbearbeitung nach Wiederinbetriebnahme" zeigt anhand eines Ablaufdiagramms die grundlegenden Entscheidungsschritte im Zuge der Auftragsbearbeitung ab dem Zeitpunkt der Wiederinbetriebnahme von Gasse 0 im leicht vereinfachten Überblick: Handelt es sich bei einem vorliegenden Auftragstyp um eine Einlagerung, weist die Lagersteuerung entsprechend der Querverteilung der Lagergasse die einzulagernde Lagereinheit zu, die über den geringsten Lagerbestand des entsprechenden Artikels verfügt. Handelt es sich beim betrachteten Auftrag um eine durchzuführende Auslagerung, bestimmt die Reihenfolge, in der die entsprechenden Lagereinheiten eingelagert wurden darüber, aus welcher Lagergasse der nachgefragte Artikel zu entnehmen ist.

Bezogen auf das gewählte Beispiel und die Situation zum Zeitpunkt der Wiederinbetriebnahme von Gasse 0 ergeben sich dabei folgende Zusammenhänge: Einlagerungen würden zunächst zwischen den Lagergassen alternierend erfolgen. Sobald es allerdings zu den ersten vier Auslagerungen des entsprechenden Artikels kommt, wird damit ausschließlich der Bestand der Gasse 0 reduziert, wodurch die folgenden vier Einlagerungen zwangsläufig aufgrund des im Vergleich zu den beiden übrigen Gassen geminderten Artikelbestandes derselben Gasse 0 zugewiesen werden. Es lässt sich also von einer Zwangsfolge sprechen, die sich aus der gassenweise ungleichen Verteilung nacheinander auszulagernder Einheiten ergibt, 
wonach die ersten erfolgten Auslagerungen aus Gasse 0, die anschließend auf sie folgenden Einlagerungen auf eben dieselbe Gasse 0 lenken.

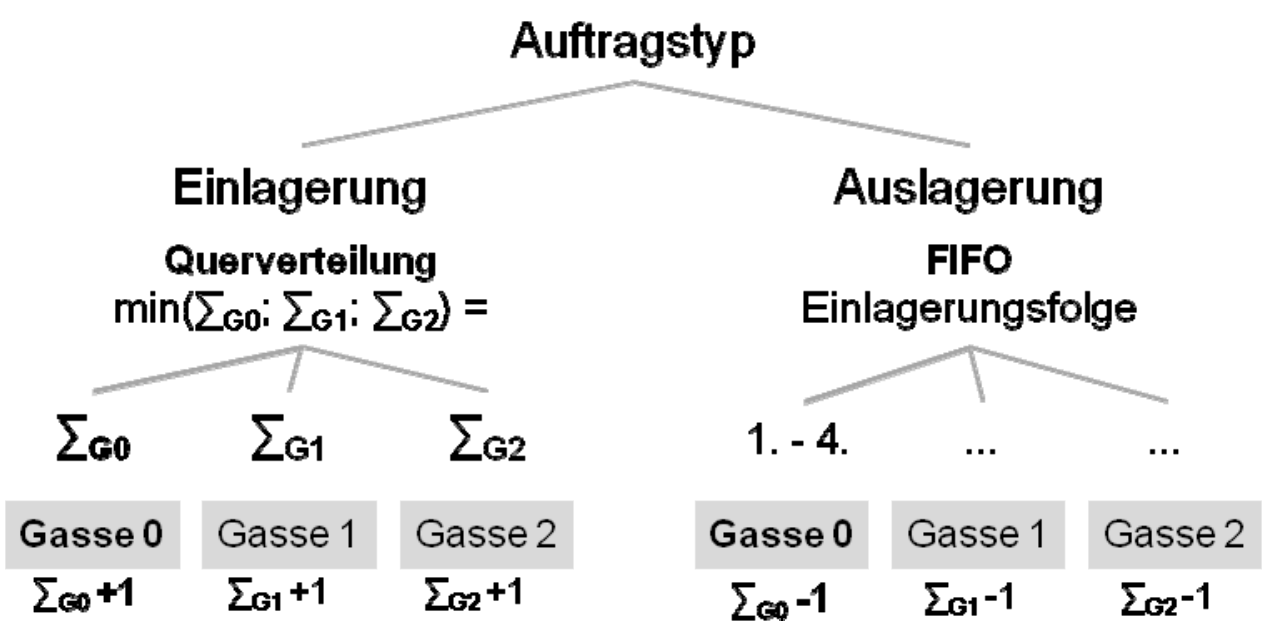

Abbildung 4: Algorithmus der Auftragsbearbeitung nach Wiederinbetriebnahme

\section{$3 \quad$ Wiederinbetriebnahme einer Gasse im Lagerbetrieb}

Die Abbildungen 1 - 4 und deren Erläuterungen betrachten alleine die Verteilung der Lagereinheiten und das Verhalten der Lagersteuerung in Bezug auf einen bestimmten Artikel. Die dargestellten Wirkungszusammenhänge bestehen allerdings darüber hinaus für alle Lagerartikel, die während und nach einem Gassenausfall einund ausgelagert werden. Folglich muss man also, aufgrund der während eines Gassenausfalls entstehenden gassenweisen Ungleichverteilung von nacheinander auszulagernden Einheiten (FIFO) und der nach Wiederinbetriebnahme der ausgefallenen Gasse auftretenden Zwangsfolge - für ausgeführte Auslagerungen entsprechend viele Einlagerungen derselben Gasse zu zuweisen (Querverteilung) eine Konzentration durchzuführender Ein- und Auslagerungen bzgl. der wieder in Betrieb genommenen Gasse erwarten.

Anhand geeigneter Experimente an einem diskreten, ereignisorientierten Simulationsmodell lässt sich nachweisen, dass der Durchsatz in Zeilenregallagern unter dem Regime der beiden Lagerbetriebsstrategien Querverteilung und FIFO nach Wiederinbetriebnahme einer vorübergehend ausgefallenen Regalgasse zunächst deutlich abfallen kann. Abbildung 5: „Variationen der Ausfalldauer 24h - 6h (geglättete Darstellung)“ [Wil10] zeigt den sich ergebenden kombinierten Auslagerdurchsatz $Q_{K A}$ nach [VDI98] in Abhängigkeit unterschiedlich langer Ausfallzeiten einer Gasse. 


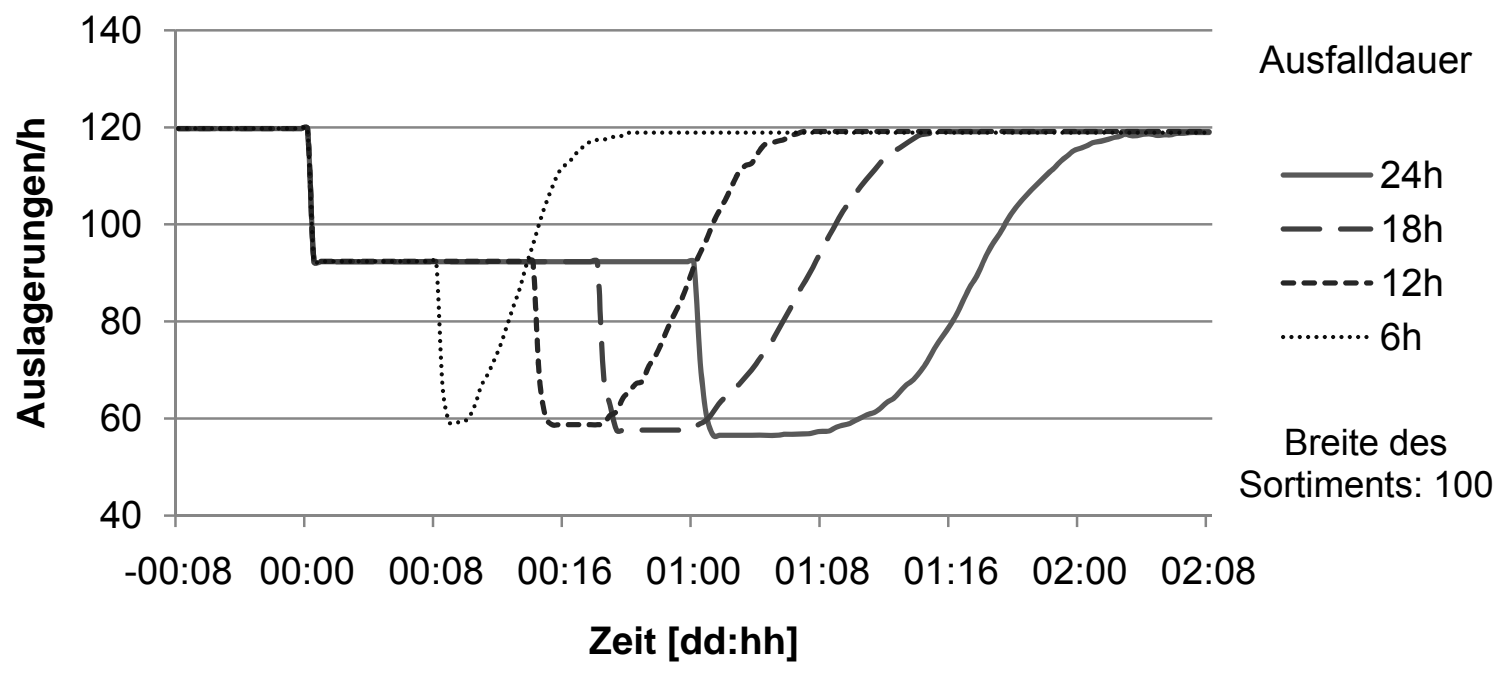

- 3 Gassen, 100 x 20 Lagerplätze pro Regal (einfach-tief), mittlerer Füllungsgrad: ca. $80 \%$

- RBG-Kenndaten: $a_{\mathrm{x}}=\mathrm{a}_{\mathrm{y}}=1,0 \mathrm{~m} / \mathrm{s}^{2} ; \mathrm{v}_{\mathrm{x}}=5,0 \mathrm{~m} / \mathrm{s} ; \mathrm{v}_{\mathrm{y}}=2,5 \mathrm{~m} / \mathrm{s}$; $\mathrm{t}_{\mathrm{z}}=2,0 \mathrm{~s}$ (vollst. Lastübergabe); $\mathrm{t}_{0}=0,75 \mathrm{~s}$

- kontinuierliche Einlastung von zufällig generierten Ein- und Auslageraufträgen (gleichverteilt hinsichtlich des Artikelsortiments)

- Betriebsstrategien: freie Lagerplatzvergabe, Ein- und Doppelspiele, Querverteilung, FIFO

- jeweils ein ,Pufferplatz، vor jeder Gasse: befindet sich darauf zum Abschlusszeitpunkt des vorhergehenden Spiels eine passende Kombination aus Ein- und Auslagerauftrag, führt das RBG ein Doppelspiel aus - andernfalls (ohne Wartezeit) ein Einzelspiel.

Abbildung 5: Variationen der Ausfalldauer 24h - 6h (geglättete Darstellung)

Anzumerken sei hierbei, dass sich das Durchsatzniveau insgesamt durchaus noch durch die Anwendung weiterer entsprechend geeigneter Betriebsstrategien, wie etwa Schnelläuferzonierung, Fahrwegoptimierung, Einrichtung weiterer Pufferplätze steigern und die Erstellung optimaler Auftragsfolgen unter den dargestellten Rahmenbedingungen anheben ließe (hierzu u.a. [Bra97], [Gra94], [Sch08]). Unabhängig davon aber ergibt sich in den betrachteten Fällen eine vorübergehende Minderung des kombinierten Durchsatzes auf den Wert, den ein einzelnes Regalbediengerät - nämlich das der zuvor ausgefallenen Gasse - alleine erzielt. Aus der uneingeschränkten Anwendung von Querverteilung und FIFO-Prinzip stellt in der betrachteten Situation eben dieses Regalbediengerät vorübergehend einen Engpass mit der Wirkung einer Leistungsdrossel [Gud10] für das gesamte Lagersystem dar.

Das dargestellte Problem des Einbruchs des maximal-realisierbaren Durchsatzes hat insbesondere vor dem Hintergrund der Planungsempfehlung Bewandtnis, die Durchsatzgrenzleistungen eines Logistiksystems so zu bemessen, dass der mittlere Jahresdurchsatz innerhalb der Normalbetriebszeit zu bewältigen ist. Die 
Durchsatzgrenzleistungen eines Logistiksystems, wie beispielsweise ein automatisches Zeilenregallager, berechnet sich hierbei aus den partiellen Grenzleistungen $\mu_{\mathrm{i}}$, die sich gemäß Formel 1 [Gud10] aus der technischen Verfügbarkeit $\eta_{\text {ver, }}$, der stochastischen Auslastbarkeit $\eta_{\text {aus, }}$, der Gerätekapazität $C_{L G}$ und der entsprechenden (mittleren) Spielzeit $\mathrm{T}_{\mathrm{i}}$ zusammensetzt.

$$
\mu_{i}=\eta_{\text {ver }} * \eta_{\text {aus }} * 3600 * \frac{C_{L G}}{\tau_{i}}
$$

Die stochastische Auslastbarkeit $\eta_{\text {aus }}$ ergibt sich hierbei laut [Gud10] aus dem schwankenden Zulauf der Ein- und Auslageraufträge und aus den variablen Spielzeiten der Lagergeräte. Zudem ist sie abhängig von der Anzahl der Pufferplätze auf dem Zu- und Abfördersystem vor und nach den Übernahme- und Übergabeplätzen des Lagerbereichs. Die Gerätekapazität $C_{L G}$ gibt an, wie viele Ladeeinheiten das betrachtete Regalbediengerät aufnehmen kann.

Sämtliche, in diesem Term (Formel 1) nicht berücksichtigten Einflüsse firmieren unter ,besondere Durchsatzanforderungen', welche durch flexible Ausdehnung der Betriebszeit abzufangen wären [Gud10] (- jedenfalls genügen die Begriffe technische Verfügbarkeit und stochastische Auslastbarkeit in ihrer bisherigen Prägung nicht, um die dargestellten Folgen ausreichend zu beschreiben).

Die beschriebenen Effekte können zunächst dazu führen, dass die geltenden Anforderungen hinsichtlich Termintreue nicht eingehalten werden können. Darüber hinaus machen sämtliche Durchsatzeinbußen, die aufgrund des Ausfalls einer Gasse über die Minderung während des Ausfalls hinaus zusätzlich entstehen, es im Normalfall notwendig, kurzfristig zusätzliche Betriebszeiten (Schichten) einzurichten, um die entstandenen Rückstände wieder aufzuholen, was allerdings keinesfalls als ein befriedigender Umgang mit der dargestellten Problematik angesehen werden kann: Für die Kalkulation der für diese zusätzlichen Betriebszeiten entstehenden Kosten muss in Betracht gezogen werden, dass nicht nur die Durchsatzminderungen aufzuholen sind, die während und im direkten Anschluss an den Ausfall einer Gasse auftreten, sondern auch solche, die sich ggf. erst verspätet bemerkbar machen.

Abbildung 6: „Zusätzliche, verzögerte Durchsatzminderungen“ (nach [Wil10]) zeigt den kombinierten Auslagerdurchsatz über die Zeit für das im Zusammenhang mit Abbildung 5 beschriebene Simulationsmodell. Ab dem Zeitpunkt 02:20 [dd:hh] zeigen sich in dieser Darstellung erneute, vorübergehende Formen von Durchsatzminderungen - nachdem das ursprüngliche Durchsatzniveau schon vor 02:00 [dd:hh] bereits wieder erreicht wurde. Diese weiteren Minderungen sind in der Tatsache begründet, dass die verstärkt vorgenommenen Einlagerungen, die in Zwangsfolge auf die Auslagerungen aus der betroffenen Gasse nach deren Wiederinbetriebnahme erfolgten, selbst wiederum auf die Gassen ungleich verteilte Auslagerungen nach sich ziehen. Die Ungleichverteilungen, die während des Ausfalls einer Gasse entstehen, sind also nicht zum Zeitpunkt des erstmaligen 
Erreichens des vormaligen Durchsatzniveaus vollständig beseitigt, sondern können durchaus weitere, verzögerte Folgeminderungen verursachen.

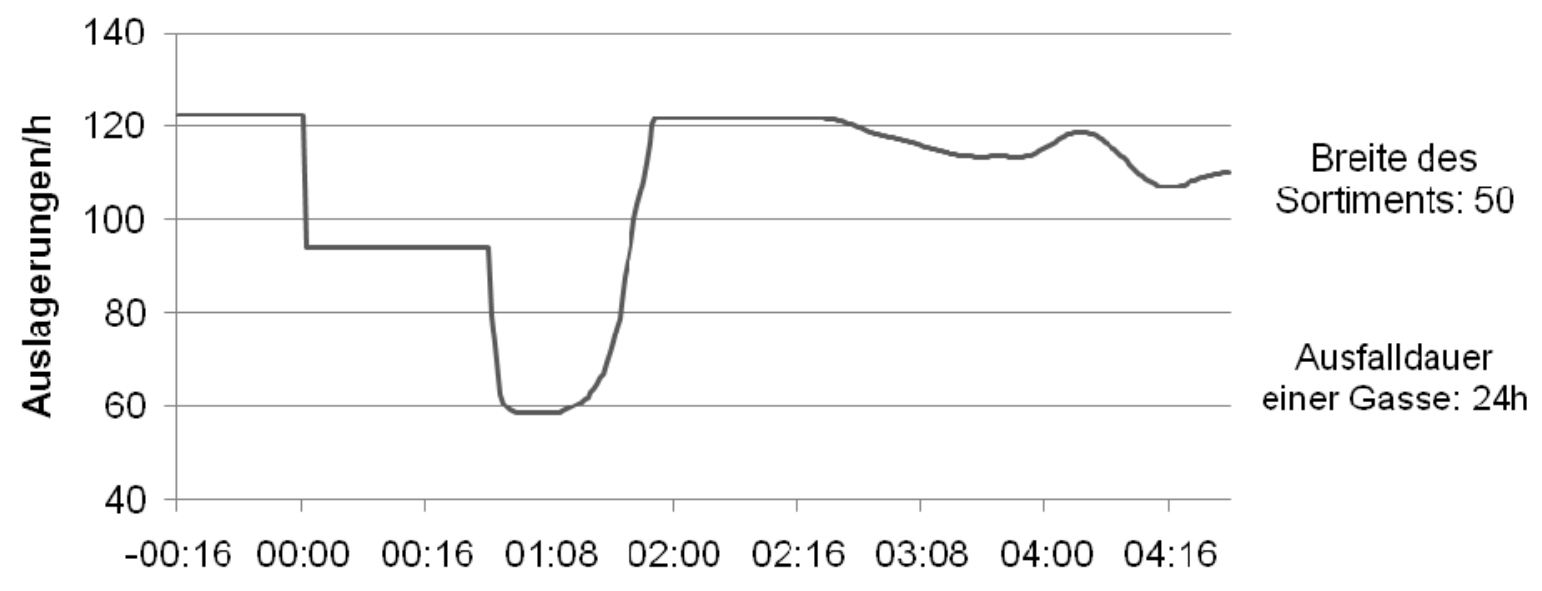

Zeit [dd:hh]

(Modell und nicht genannte Parameter identisch zu Abbildung 5)

Abbildung 6: zusätzliche, verzögerte Durchsatzminderungen (nach [Wil10])

Muss also in Folge eines Gassenausfalls die Betriebszeit ausgedehnt werden, so wären diese, möglicherweise noch nicht eingetretenen Folgeminderungen entweder auch zu antizipieren oder durch gezielte Umlagerungen zu verhindern.

Mittels Umlagerungen den im direkten Anschluss an den Ausfall einer Lagergasse folgenden Durchsatzeinbruch zu verhindern, stellt in diesem Zusammenhang auch eine Handlungsalternative dar, die allerdings hinsichtlich ihres zeitlichen Aufwands aufgrund der notwendigen Anzahl durchzuführender Umlagerungen nicht unterschätzt werden sollte [Sch10].

An der Erhöhung der Verfügbarkeit von Hochregallagersystemen wird darüber hinaus beständig gearbeitet (- beispielhaft: [Vog04], [Alt92]). Jegliche Maßnahme zur Erhöhung der Verfügbarkeit leistet einen Beitrag zur Minderung des dargestellten Problems. Hierunter lassen sich allgemein Maßnahmen zur Instandhaltung ebenso aufführen, wie auch die Einrichtung redundanter Systemelemente, was aus Kostengründen schwierig umzusetzen ist, da für die Auslegung automatischer Hochregallager die Maßgabe besteht, die Anzahl vorhandener Regalbediengeräte nach der Anzahl der Gassen zu richten [Gud10]. Die Einrichtung einer gewissen Teilredundanz ließe sich alternativ mittels Regalbediengeräten realisieren, deren Funktionsumfang die Fähigkeit zur Ausübung von Gangwechsel (Kurvengängigkeit) vorsieht - deren Einsatz allerdings nur bei geringem Lagerumschlag empfohlen wird [Gud10]. 


\section{$4 \quad$ Entwicklung neuer Handlungsoptionen}

Ein wichtiger Ansatzpunkt zur Entwicklung weiterer Handlungsoptionen muss in diesem Zusammenhang das Überdenken des Einsatzes der beiden Betriebsstrategien Querverteilung und FIFO sein.

Was die Querverteilungsstrategie anbelangt, so verspricht ihre Anwendung zwei positive Effekte, von welchen einer in der Möglichkeit zur schnellen (weil parallelen) Auslagerung von Lagereinheiten eines Artikels besteht. Hierbei muss hinterfragt werden, inwiefern besondere Anforderungen an die Durchlaufzeit entsprechender Aufträge im Vergleich zu anderen tatsächlich bestehen, ob nicht anstelle der Querverteilung andere Maßnahmen zur Durchsatzsteigerung in Frage kommen oder ob die Leistungsfähigkeit des Lagersystems nicht ein vorübergehendes Aussetzen dieser Strategie tolerieren würde.

Der zweite positive Effekt, den man sich von der Anwendung der Querverteilungsstrategie verspricht, besteht in einer Erhöhung der Zugriffsicherheit. Gemeint ist damit die Tatsache, dass Lagereinheiten eines Artikels auch während eines Gassenausfalls ausgelagert werden können, weil der Bestand eines Artikels gleichmäßig auf alle Gassen verteilt eingelagert wurde. Wichtig ist es allerdings hierbei zu beachten, dass zur Gewährleistung der Lieferfähigkeit während auftretender Anlagenausfälle alleine die Unterbrechungsreserve (eiserner Bestand) vorgesehen ist. Im Zusammenhang mit Gassenausfällen muss die Unterbrechungsreserve auf alle Gassen querverteilt vorgesehen werden. Kommt es zu einem Gassenausfall, müssen die Bestände der Unterbrechungsreserve der funktionstüchtigen Gassen die auftretenden Nachfragen decken. Werden diese Bestände während eines Gassenausfalls angebrochen, müssen sie schnellstmöglich wieder aufgefüllt werden. Eine Querverteilung weiterer Einlagerungen ist darüber hinaus nicht notwendig. Einschränkend sei in diesem Zusammenhang zudem darauf hingewiesen, dass jegliche Formen von Unterbrechungsreserven dann schwierig zu realisieren sind, wenn sich die Wertminderungsrate aufgrund Alterung oder Verderblichkeit (s.u.) hoch im Vergleich zum Artikelumschlag bzw. zur Artikeldrehzahl verhält.

Hinsichtlich der FIFO-Strategie sollte die Frage geklärt werden, in wie weit eine ausnahmslose, ,strenge' Form dieser Auslagerstrategie tatsächlich auch dann begründet ist, wenn Warehousemanagement- und Lagerverwaltungssysteme im Betrieb für eingelagerte Einheiten eine vorzusehende spezifische maximale

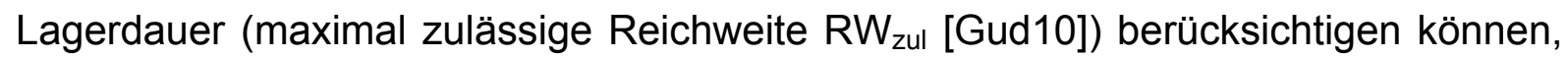
womit sich auch das Entstehen von ,Ladenhütern' ohne weiteres vermeiden ließe. Alterung und eine mögliche Verderblichkeit können möglicherweise das Absatzrisiko der Lagereinheiten erhöhen. In solchen Fällen sollte ein angemessener Risikozins kalkulatorisch in der Bestandsdisposition berücksichtigt werden [Gud10]. Beispielhaft kann für modische Waren und andere Artikel mit zeitlich begrenzter Verkäuflichkeit, wie Computer, zwischen 10 und $15 \%$ p. a. und für Artikel mit mehrjähriger 
Verkäuflichkeit und Einsetzbarkeit zwischen 3 und 5\% p. a. angesetzt werden [Gud10].

Dient ein betrachtetes automatisches Zeilenregallager zur Verwaltung von PushBeständen, lassen sich gewisse Handlungsoptionen zusammenfassend formulieren. Push-Bestand meint in diesem Zusammenhang, dass der jeweilige Bestand entweder in einem Schub eingelagert und nach einer festen Lagerdauer in gleicher Menge wieder ausgelagert oder in mehreren Schüben aufgebaut und zu einem bestimmten Zeitpunkt vollständig abgebaut oder in einem Schub eingelagert und nach kurzer Zeit in wenigen Schüben ausgelagert wird [Gud10]. Ein besonderes Merkmal dieser Bestände besteht darin, dass die genaue Lagerdauer eines PushBestands häufig bereits zum Zeitpunkt der Einlagerung bekannt oder absehbar ist [Gud10].

Hieraus ergibt sich die Möglichkeit, nach dem Ausfall einer Gasse zunächst von der Querverteilungsstrategie abzuweichen, um das entsprechende Regalbediengerät zu entlasten. Dieses kann dann falls nötig alleine dazu eingesetzt werden, anstehende Auslagerungen aus der wieder in Betrieb genommenen Gasse durchzuführen. Rechtzeitig vor dem absehbaren Auslagerungstermin sollte dann aber mittels Umlagerungen eines Teils der Einheiten wieder eine Querverteilung hergestellt werden.

Was anstehende Auslagerungen betrifft, so lässt sich ggf. das ,strenge‘ FIFO-Prinzip hin zu einer ,schwachen' FIFO-Strategie aufweichen [Gud10], was bedeuten würde, dass nur die Zugehörigkeit zu einer Einlagerungs-Charge bei der Bestimmung der Auslagerungsreihenfolge zu beachten wäre. Soweit bis zum anstehenden Auslagerungstermin entsprechend Zeit zur Verfügung steht, sollten die Bestände der vormals ausgefallen Gasse rechtzeitig auf alle Gassen des Lagers verteilt werden andernfalls ergibt sich ggf. in einer deutlicheren Abweichung vom FIFO-Prinzip die Möglichkeit, die Zusammenstellung anstehender Auslagerungsaufträge gezielt zu streuen [Wil10].

Darüber hinaus sollten sich die zu ergreifenden Maßnahmen an die im Einzelfall zu verifizierende Risikolage anpassen: d.h. an die technische Verfügbarkeit der Lagergassen, die Folgen von möglichweise auftretenden Verzügen von Lieferterminen, der Wertminderung aufgrund Alterung oder Verderblichkeit, die Betriebskosten sowie die aktuelle und vorhersehbare Auslastung der Anlagen.

\section{Literatur}

[Alt92] Alt, M.: Bei Ausfall der DV steht das Lager - welche Möglichkeiten es gibt, den Ernstfall zu vermeiden. In: Logistik im Unternehmen; Bd. 6, 1992, Nr. 3; S. 44-47

[Bra97] Brandes, T.: Betriebsstrategien für Materialflusssysteme unter besonderer Berücksichtigung automatisierter Lager. Aachen: Shaker 1997 
[Gei98] Geinitz, J.: Unerkannte Abhängigkeiten mindern die Leistungsfähigkeit automatisierter Lager. In: Marktbild Lager, Bd. 18, 1998, S. 16-18

[Gra94] Grafe, W.: Gestaltung und Betrieb von Lagersystemen mit hohen Umschlagleistungen. In: Jünemann, R. (Hrsg.): Logistik für die Praxis. Dortmund: Praxiswissen 1994

[Gud06] Gudehus, T.: Dynamische Disposition : Strategien zur optimalen Auftrags- und Bestandsdisposition. 2. Auflage, Berlin, Heidelberg: Springer 2006

[Gud10] Gudehus, T.: Logistik: Grundlagen - Strategien - Anwendungen. 4. Auflage, Berlin, Heidelberg: Springer 2010

[Ker96] Kern, W. (Hrsg.): Handwörterbuch der Produktionswirtschaft. In: Enzyklopädie der Betriebswirtschaftslehre: Bd. 7. 2. Auflage, Stuttgart: Schäffer-Poeschel 1996

[Mar06] Martin, H.: Transport- und Lagerlogistik : Planung, Struktur und Kosten von Systemen der Intralogistik. 6. Auflage, Wiesbaden: Vieweg 2006

[Sch08] Schumann, M.: Zur Bestimmung der Umschlagleistung von Hochregallagern unter besonderer Berücksichtigung der Lagerorganisation. Chemnitz, Dissertation, 2008

[Sch10] Scheid, W.-M.: Havarie bei Anlagen der Intralogistik. In: WolfKluthausen, H. (Hrsg.): Jahrbuch Logistik, Bd.24, Korschenbroich: free beratung 2010, S. 30-33

[tS08] ten Hompel, M.; Schmidt, T.: Warehouse Management : Organisation und Steuerung von Lager- und Kommissioniersystemen. 3. Auflage, Berlin, Heidelberg: Springer 2008

[tSN07] ten Hompel, M.; Schmidt, T.; Nagel, L.: Materialflusssysteme : Förderund Lagertechnik. 3. Auflage, Berlin, Heidelberg: 2007

[VDI98] Verein Deutscher Ingenieure VDI: Richtlinie 4480, Blatt 1 - Durchsatz von automatischen Lagern mit gassengebundenen Regalbediensystemen. Berlin: Beuth, 1998

[Vog04] Vogt, M.: Sehende Regalbediengeräte. In: Fördertechnik : Zeitschrift für Logistik, Materialfluss, Transport- und Lagertechnik; Bd. 73, 2004, Nr. 78, S. $30-31$

[Will10] Wildner, C.: Anlaufstrategien für Hochregallager bei Wiederinbetriebnahme eines ausgefallenen Regalbediengeräts, in: Wissenschaftliche Gesellschaft für Technische Logistik: Tagungsband 6. Fachkolloquium der Wissenschaftlichen Gesellschaft für Technische Logistik (WGTL), Garbsen: PZH Produktionstechnisches Zentrum (2010), S. 163-174 